\title{
Comparison of local recurrence in transcatheter arterial chemoembolization of hepatocellular carcinoma with or without accumulation of iodized oil beyond corona enhancement area: Short-term results
}

\author{
Yukinobu Watanabe, Masahiro Ogawa, Masahiro Kaneko, Mariko Kumagawa, \\ Midori Hirayama, Naoki Matsumoto, Hiroshi Nakagawara, Toshiki Yamamoto, \\ Mitsuhiko Moriyama
}

\author{
Department of Gastroenterology and Hepatology, Nihon University School of Medicine, Tokyo, Japan \\ Radiol Oncol 2022; 56(1): 69-75. \\ Received 12 August 2021 \\ Accepted 1 October 2021 \\ Correspondence to: Prof. Yukinobu Watanabe, Department of Gastroenterology and Hepatology, Nihon University Hospital, 1-6 Kanda, \\ Surugadai, Chiyoda-ku, Tokyo 101-8309, Japan. E-mail: koushin0809@yahoo.co.jp \\ Disclosure: No potential conflicts of interest were disclosed. \\ This is an open access article under the CC BY-NC-ND license (http://creativecommons.org/licenses/by-nc-nd/4.0/).
}

Background. Local tumor recurrence of hepatocellular carcinoma (HCC) often occurs in blood drainage areas. Corona enhancement is determined by computed tomography during hepatic arteriography (CTHA) and is considered to represent the blood drainage area. This study aimed to investigate the relationship between embolization of corona enhancement area and local tumor recurrence of patients with HCC who underwent transcatheter arterial chemoembolization (TACE).

Patients and methods. The study retrospectively selected 53 patients with $60 \mathrm{HCC}$ nodules that showed corona enhancement area on late-phase CTHA and showed homogenous accumulation of iodized oil throughout the nodule on non-contrast-enhanced CT performed immediately after TACE. We divided the nodules into two groups, according to whether the accumulation of iodized oil covered the entire corona enhancement area (group A) or not (group B). Local tumor recurrence was compared between the two groups.

Results. The cumulative local tumor recurrence rates for group A $(n=36)$ were $2.8 \%, 2.8 \%, 8.3 \%$ at 3,6 , and 12 months, respectively, whereas the recurrence rates for group $B(n=24)$ were $20.8 \%, 45.8 \%, 75 \%$ at 3,6 , and 12 months, respectively. The cumulative local tumor recurrence rates for group A were significantly lower than those for group $B$ (hazard ratio, 0.079; 95\% confidence interval, 0.026-0.24; $p<0.001$ ).

Conclusions. The results of the study suggest that the corona enhancement area may be an accurate safety margin in TACE which should be performed until the embolic area covers the entire corona enhancement area.

Key words: corona enhancement; transcatheter arterial chemoembolization; computed tomography during hepatic arteriography; hepatocellular carcinoma

\section{Introduction}

Hepatocellular carcinoma (HCC) is the most common primary liver malignancy and is the third leading cause of cancer death worldwide. ${ }^{1}$ Transcatheter arterial chemoembolization (TACE) has been widely used to treat unresectable HCC. ${ }^{2-5}$ According to the Barcelona Clinic Liver Cancer staging system, TACE is the first-line treatment for patients with intermediate-stage $\mathrm{HCC}^{6}$, underlining the importance of TACE in the treatment of HCC. 
HCC often has satellite lesions that cannot be diagnosed by imaging modalities, and the local tumor recurrences may occur because of untreated satellite lesion.,8 Therefore, it is necessary to treat not only the tumor itself but also the area around the tumor in the treatment of HCC. Previous studies reported that the adequate safety margin of iodized oil in TACE also affects microsatellite lesions around the tumor, which resulted in a lower rate of local tumor recurrence. ${ }^{9-11}$

Corona enhancement is one of the characteristic findings of hypervascular HCC, and it results in perinodular enhancement with bright branching structures depicted on late-phase computed tomography during hepatic arteriography (CTHA). ${ }^{12-16}$ The corona enhancement area has been proposed as the blood drainage area of HCC and a previous study reported that satellite lesions of HCC existed in the blood drainage area. ${ }^{17}$ The corona enhancement area is expected to be an accurate safety margin in the treatment of HCC. However, there have been no studies that have evaluated the association between embolization of corona enhancement area and local tumor recurrence following TACE.

The aim of this retrospective study was to investigate the relationship between local recurrence following TACE and embolization of corona enhancement area.

345 Patients treated with TACE

\section{Number of excluded patients} performed DEB-TACE $n=20$ more than 5 nodules $\mathrm{n}=136$ extrahepatic metastases $\mathrm{n}=1$ impaired renal function $\mathrm{n}=5$ not performed CTHA $n=61$

122 patients 178 nodules treated with TACE

Number of excluded nodules prior treatment $n=48$ no corona enhancement $\mathrm{n}=35$ insufficient accumulation of iodized oil $\mathrm{n}=35$

53 patients 60 nodules treated with TACE

FIGURE 1. Flow diagram of patients' and nodules' selection.

$\mathrm{CTHA}=$ computed tomography during hepatic arteriography; $\mathrm{DEB}-\mathrm{TACE}=$ drug-eluting beads transcatheter arterial chemoembolization; RFA = radiofrequency ablation; TACE = transcatheter arterial chemoembolization

\section{Patients and methods}

This study was conducted in accordance with the guidelines of the Declaration of Helsinki and was approved by our institutional ethics committee (protocol number 20200502). Informed consent was waived by the committee because this study was a retrospective study.

\section{Study population}

Between April 2016 and October 2019, TACE procedures were performed on 345 patients with HCC at our hospital. TACE was done because patients were ineligible for surgery or they refused to undergo surgical resection. Radiofrequency ablation (RFA) procedures were not performed due to the location of the tumor, where ablation may cause insufficient therapeutic effects and/or adverse effects on adjacent organs. The diagnosis of HCC was confirmed by at least 2 of the following modalities: contrast-enhanced computed tomography (CECT), contrast-enhanced ultrasonography, and/or gadolinium-ethoxybenzyl-diethylenetriamine pentaacetic acid (Gd-EOB-DTPA)-enhanced magnetic resonance imaging (MRI). Eligibility criteria for this study were as follows: (a) the presence of fewer than five nodules; (b) nodules treated with balloonoccluded TACE (B-TACE); (c) nodules that showed corona enhancement on late-phase CTHA; and (d) nodules showing dense accumulation of iodized oil throughout the tumor on non-contrast-enhanced CT performed immediately after TACE. The exclusion criteria were as follows: (a) the nodules with prior treatment; (b) impaired renal function, a contraindication for CECT; and (c) extrahepatic metastases. Fifty-three patients with a total of 60 nodules were enrolled in this study. A flow diagram of patient and nodule selection is shown in Figure 1.

The nodules were retrospectively divided into two groups based on the degree of iodized oil accumulation identified on non-contrast-enhanced CT performed immediately after TACE. In group $\mathrm{A}$, a dense accumulation of iodized oil covered the whole tumor and the entire corona enhancement area. In group B, a dense accumulation of iodized oil covered the whole tumor but did not cover the entire corona enhancement area.

\section{CTHA procedure}

Double-phase CTHA was performed in all patients prior to TACE. After left brachial artery puncture, A 4-Fr catheter (FNSAC IV, Angiomaster; Terumo, 
Tokyo, Japan) was selectively inserted into the proper, common, or replaced hepatic artery and CTHA was performed to identify tumor staining, corona enhancement, and the feeding arteries of the tumor. Examinations were performed using a 64 multidetector-row CT scanner (Aquilion CX; Canon, Tokyo, Japan). The imaging CT parameters were rotation time $0.5 \mathrm{~s}$, beam collimation $64 \times 0.5$ $\mathrm{mm}, 0.5 \mathrm{~mm}$ slice thickness at $0.4 \mathrm{~mm}$ intervals, a tube voltage $120 \mathrm{kV}$, and volume EC. Helical scanning was initiated 6 or $7 \mathrm{~s}$ after the infusion of 13 $\mathrm{ml}$ iomeprol (iodine concentration $350 \mathrm{mg} / \mathrm{mL}$; Iomeron, Eisai, Tokyo, Japan) diluted with $26 \mathrm{ml}$ of saline into the common, proper, or replaced hepatic artery at a rate of 3 or $3.5 \mathrm{ml} / \mathrm{s}$. The late-phase scanning was performed $40 \mathrm{~s}$ after the initiation of the infusion.

\section{TACE procedure}

All patients had a B-TACE using a 1.8-Fr microballoon catheter (Attendant Delta, Terumo, Tokyo, Japan) through a 4-Fr catheter. The microballoon catheter was placed as close to the tumor feeding artery as possible, and miriplatin (MIRIPLA; Dainippon-Sumitomo Pharmaceutical, Tokyo, Japan) suspended in iodized oil (Ultra-Fluid; Dainippon-Sumitomo Pharmaceutical) was injected into the tumor feeding artery under balloon occlusion. The doses of miriplatin were determined on the basis of tumor size, however. injections of miriplatin were stopped immediately before the flow ceased completely. The total amount of miriplatin per session was limited to $120 \mathrm{mg}$. This stage was followed by embolization with 1-mm gelatin sponge particles (Gerpart; Nippon Kayaku, Tokyo, Japan) crushed by pumping ten times with two 2.5$\mathrm{mL}$ syringes and a three-way stopcock. If several tumor feeding arteries were confirmed, B-TACE was also performed through each artery.

\section{Image evaluation}

Double-phase CTHA images were evaluated to determine whether corona enhancement was depicted around the hypervascular tumor. When intranodular enhancement was observed on the early-phase CTHA and subsequently perinodular enhancement appeared on the late phase of CTHA, we determined that corona enhancement was positive in this study. we also measured the thickness of corona enhancement. Based on previous report $^{16}$, perinodular enhancement with or without irregular protrusions that was greater than $2 \mathrm{~mm}$ thickness was classified as thick corona enhancement, and flat perinodular enhancement that was less than or equal to $2 \mathrm{~mm}$ thick was classified as thin corona enhancement. In all patients, an unenhanced CT was performed immediately after TACE to check for iodized oil accumulation in the target nodules and the nodules were divided into group A or B based on the degree of iodized oil accumulation. Contrast-enhanced CT was performed 4 weeks after TACE for evaluation of the treatment effect. If no local tumor recurrence was identified, then contrast-enhanced CT or Gd-EOB-DTPAenhanced MRI was performed every 3-4 months thereafter. Local tumor recurrence was judged when identifiable nodular enhancement in the arterial phase was seen in or adjacent to the treated tumor. In this study, two board-certified hepatologists (> 10 years of experience in abdominal CT) independently assessed the images of unenhanced $\mathrm{CT}$, contrast-enhanced CT, Gd-EOB-DTPA enhanced MRI, and CTHA. If two hepatologists had different assessments, the final result of that particular investigation was obtained by consensus through discussion of them.

\section{Statistical analysis}

Data analyses were performed using EZR (Saitama Medical Center, Jichi Medical University, Saitama, Japan), a graphical user interface for $R$ (The $R$ Foundation for Statistical Computing, Vienna, Austria).

The significance of differences in background parameters was evaluated by the Mann-Whitney U test and Fisher exact test. Univariate and multivariate logistic regression analyses were performed to identify the factors correlated with local tumor recurrence. The main objective of this study was to investigate the relationship between embolization including corona enhancement area and local tumor recurrence in TACE, and thus, tumor recurrence rates were compared between group $\mathrm{A}$ and group B. The cumulative local recurrence rate was calculated using the Kaplan-Meier method and evaluated using the log-rank test. $p$ values of less than 0.05 were considered to be a statistically significant difference.

\section{Results}

Group A had 36 cases and group B had 24 cases. The clinical characteristics of nodules are summarized in Table 1. Except for des-gamma-carboxy 
TABLE 1. Clinical characteristics of nodules in group $A$ and $B$

\begin{tabular}{lccc}
\hline Clinical characteristics & Group A $(\mathbf{n}=\mathbf{3 6})$ & Group B $(\mathbf{n}=\mathbf{2 4})$ & p value \\
\hline Age, years* & $74(70-79)$ & $71(64-79)$ & 0.149 \\
\hline Gender, male/female & $8 / 28$ & $6 / 18$ & 0.999 \\
\hline Etiology (HCV/HBV/Alcohol/Unknown) & $16 / 8 / 8 / 4$ & $9 / 5 / 6 / 4$ & 0.912 \\
\hline Child-Pugh classification (A/B) & $31 / 5$ & $19 / 5$ & 0.569 \\
\hline BCLC stage(O/A/B) & $11 / 20 / 5$ & $5 / 17 / 2$ & 0.545 \\
\hline Size of tumor, mm* & $14.5(12-21.3)$ & $15.5(11-22)$ & 0.757 \\
\hline Thickness of corona enhancement( $\leq 2 \mathrm{~mm} />2 \mathrm{~mm})$ & $17 / 19$ & $10 / 14$ & 0.793 \\
\hline AFP, ng/ml* & $4.9(2.6-10.6)$ & $5.9(2.7-14.1)$ & 0.419 \\
\hline DCP, mAU/mL* & $26.4(17.0-49.2)$ & $48.8(25.3-153.9)$ & 0.021 \\
\hline Usage of miriplatin, mg* & $28(20-38.5)$ & $30(22.4-51.5)$ & 0.310 \\
\hline
\end{tabular}

AFP = alpha-fetoprotein; $B C L C=$ The Barcelona Clinic Liver Cancer Classification; $D C P=$ des-gamma-carboxy prothrombin; HBV = hepatitis B virus; HCV $=$ hepatitis $\mathrm{C}$ virus;

* = data are the median (interquartile range)

prothrombin, the baseline characteristics did not differ significantly between groups A and B.

In the total sample, local tumor recurrence was observed in 22 out of 60 cases $(36.7 \%)$ and the cumulative 3-, 6-, 12-month tumor local recur-

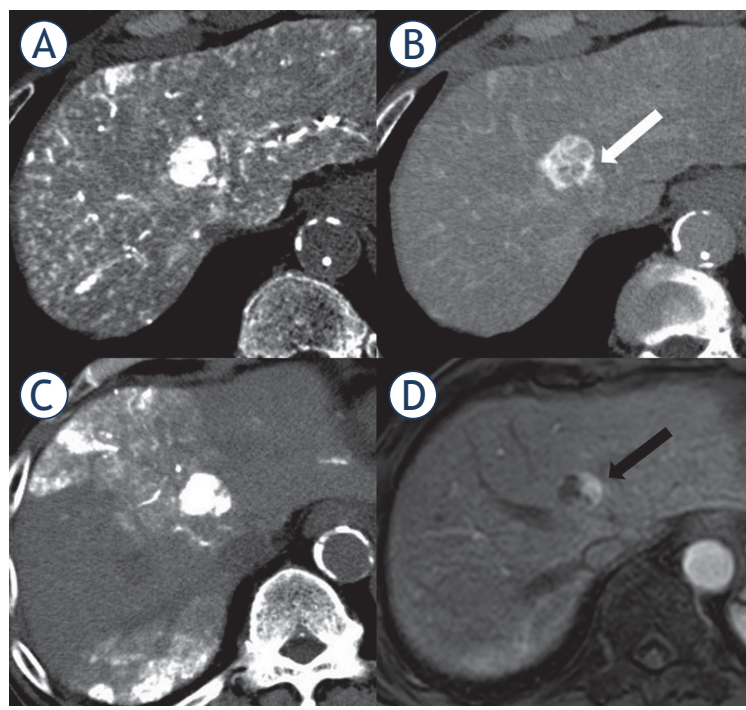

FIGURE 2. Local tumor recurrence after transcatheter arterial chemoembolization (TACE) in an 82-year-old patient with a hepatocellular carcinoma. (A) Early-phase computed tomography during hepatic arteriography (CTHA) demonstrated a hypervascular nodule in S5. (B) Late-phase CTHA demonstrated the corona enhancement around the tumor (white arrow). (C) Non-contrast-enhanced computed tomography performed immediately after TACE showed dense accumulation of iodized oil throughout the tumor, but not in the entire corona enhancement area. (D) In contrast enhanced magnetic resonance images obtained 4 months after TACE, local recurrence developed around the tumor (black arrow). rence rates for all cases were $10 \%, 20 \%$, and $35 \%$ respectively. The median time to local recurrence was 167 days (range, 32-375 days). In group B, local tumor recurrence was observed in 18 out of 24 cases (75\%) (Figure 2). Meanwhile, in group A, lo-

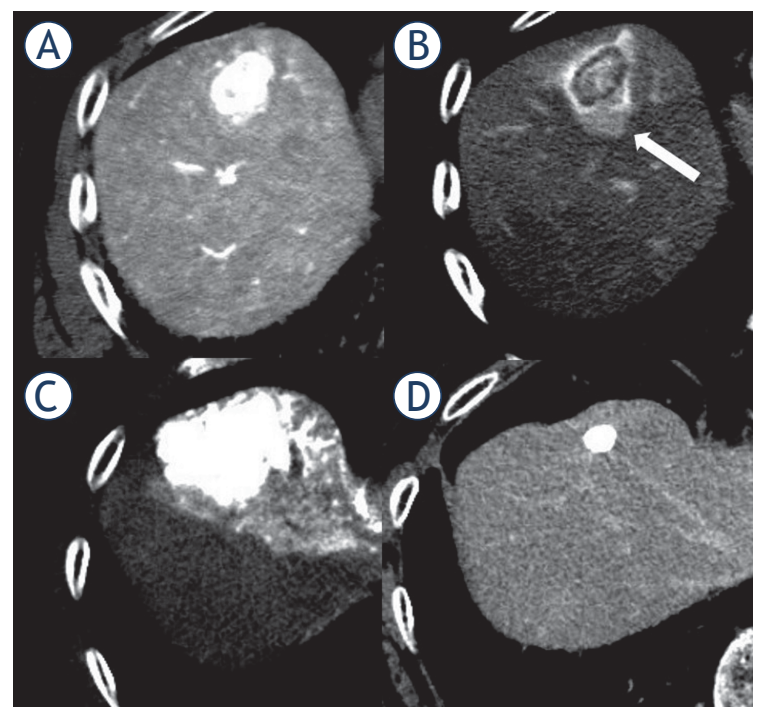

FIGURE 3. Complete response after transcatheter arterial chemoembolization (TACE) in a 77-year-old patient with a hepatocellular carcinoma. (A) Early-phase computed tomography during hepatic arteriography (CTHA) demonstrated a hypervacular nodule in S4. (B) Late-phase CTHA demonstrated the corona enhancement around the tumor (white arrow). (C) Non-contrast-enhanced computed tomography performed immediately after TACE showed dense accumulation of iodized oil beyond corona enhancement area. (D) Contrast-enhanced computed tomography performed 15 months after TACE showed no enhancement around the tumor. 
TABLE 2. Univariate and multivariate logistic regression analysis for factor affecting local tumor recurrence

\begin{tabular}{|c|c|c|c|c|}
\hline \multirow{2}{*}{ Factor } & \multicolumn{2}{|c|}{ Univariate analysis } & \multicolumn{2}{|c|}{ Multivariate analysis } \\
\hline & Odds ratio $(95 \% \mathrm{Cl})$ & $p$ value & Odds ratio $(95 \% \mathrm{Cl})$ & $p$ value \\
\hline Size of tumor (<20 vs. $\geq 20 \mathrm{~mm}$ ) & $0.67(0.20-2.32)$ & 0.577 & & \\
\hline $\operatorname{AFP}(<10 \mathrm{vs} . \geq 10 \mathrm{ng} / \mathrm{mL})$ & $0.45(0.13-1.61)$ & 0.242 & & \\
\hline $\mathrm{DCP}(<40 \mathrm{vs} . \geq 40 \mathrm{mAU} / \mathrm{mL}$ ) & $0.59(0.18-1.92)$ & 0.417 & & \\
\hline Usage of miriplatin (<30 vs. $\geq 30 \mathrm{mg}$ ) & $0.70(0.17-1.89)$ & 0.420 & & \\
\hline Thickness of corona enhancement ( $\leq 2 \mathrm{~mm} />2 \mathrm{~mm}$ ) & $0.97(0.30-3.20)$ & 0.999 & & \\
\hline Degree of iodized oil accumulation (Group A vs. B) & $0.045(0.0080-0.20)$ & $<0.001$ & $0.042(0.010-0.17)$ & $<0.001$ \\
\hline
\end{tabular}

AFP = alpha-fetoprotein; $\mathrm{Cl}=$ confidence interval; $\mathrm{DCP}=$ des-gamma-carboxy prothrombin

cal tumor recurrence was observed in only 4 out of 36 cases $(11.1 \%)$, and most nodules had no local tumor recurrence (Figure 3). The cumulative 3-, 6-, 12-month tumor local recurrence rates were $2.8 \%$, $2.8 \%, 8.3 \%$, respectively for group $\mathrm{A}$, and $20.8 \%$, $45.8 \%, 75 \%$, respectively for group B. The cumulative local tumor recurrence rates in group A were significantly lower than those in group B (hazard ratio $[\mathrm{HR}], 0.079 ; 95 \%$ confidence interval $[\mathrm{CI}]$, $0.026-0.24 ; p<0.001$ ) (Figure 4).

Univariate logistic regression analysis for prognostic factors affecting local tumor recurrence was performed for the following factors: the size of tumors, serum alpha-fetoprotein level, serum des-gamma-carboxy prothrombin level, usage of miriplatin, thickness of corona enhancement, and the degree of iodized oil accumulation (Group A or B). The degree of iodized oil accumulation only showed $p$ values $<0.05$ in univariate analysis. Multivariate analysis identified the degree of iodized oil accumulation as independent prognostic factors of local tumor recurrence $(p<0.001)$ (Table 2).

\section{Discussion}

In this study, we investigated whether the embolization of not only the tumor but also the corona enhancement area could provide a better therapeutic effect for TACE. Our findings showed that the group with a dense embolization of HCC including the corona enhancement area had a significantly lower local tumor recurrence rate and a higher therapeutic effect than the group with a dense embolization of HCC itself but not including the corona enhancement area. Our study demonstrated a relationship between local recurrence following TACE and embolization of corona enhancement area.

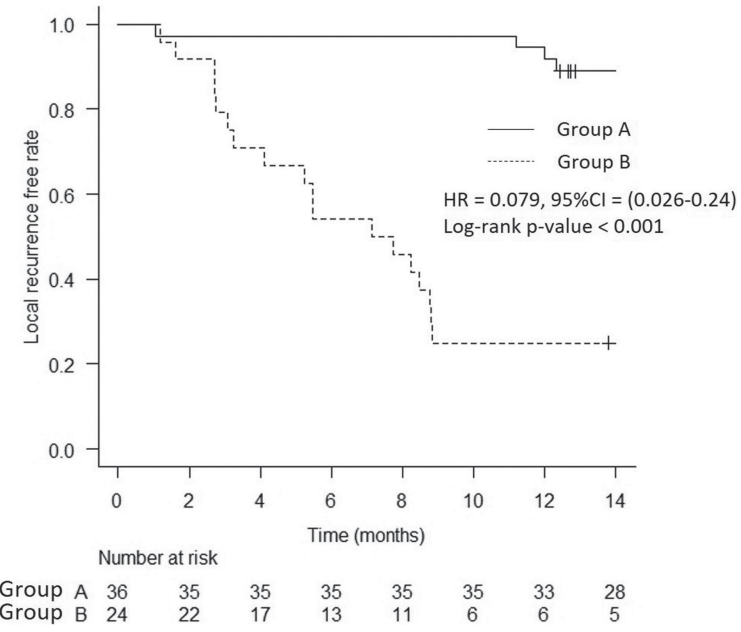

FIGURE 4. Local tumor recurrence-free rate according to the range of the embolization for transcatheter arterial chemoembolization (TACE). The cumulative 3-, 6-, 12-month tumor local recurrence rates were $2.8 \%, 2.8 \%, 8.3 \%$, respectively, for cases with embolization of the entire corona enhancement area, and $20.8 \%, 45.8 \%, 75 \%$, respectively, for cases without embolization of the entire corona enhancement area (hazard ratio $[\mathrm{HR}], 0.079 ; 95 \%$ confidence interval $[\mathrm{Cl}], 0.026-0.24 ; p<$ $0.001)$.

HCC often has satellite lesions that cannot be diagnosed by imaging modalities. ${ }^{78}$ Previous histopathological research reported that microsatellite lesions found in $46 \%$ of HCCs were smaller than 5 $\mathrm{cm}$, and in $29 \%$ of HCCs, the lesions were smaller than $2.5 \mathrm{~cm} .{ }^{18}$ Another study reported that 28 of 149 resected specimens exhibited microsatellite lesions. $^{7}$ These reports indicate that it is important to embolize the tumor and the surrounding tissues by TACE. Miyayama et al. reported that local tumor recurrence developed in $29.1 \%$ of the sufficient margin group and $66.7 \%$ of the insufficient margin group after TACE, and embolization of the safety margin was important for local tumor control by 
TACE. ${ }^{9}$ Several other studies also reported that the safety margin of iodized oil during TACE resulted in a lower rate of local tumor recurrence. ${ }^{10,11,19}$ Corona enhancement area determined by CTHA was reported to reflect the blood drainage area of $\mathrm{HCC}^{16,20}$, and this finding served as the basis for this study. We considered that the corona enhancement area would be an accurate safety margin for TACE. Hirooka et al. reported that the cumulative local recurrence rate was significantly lower in the group that ablated the entire blood drainage area, depicted as the area of corona enhancement on CTHA, than in the group that did not by RFA $(0$, 0 , and $1.5 \%$ vs. $3.8,17.0$, and $22.8 \%$ at 1,3 , and 5 years). ${ }^{21}$ Although there are differences in the procedures of RFA and TACE, the results of this previous study are similar to those of our study, indicating that the corona enhancement area can be an accurate margin for HCC treatment. The thicker the corona enhancement, the larger the drainage area that requires embolization, but thickness of corona enhancement wasn't identified as independent prognostic factors of local tumor recurrence in this study. Therefore, we consider that the size of the drainage area cannot be a factor that makes embolization of the entire drainage.

The usefulness of CTHA, including cone-beam CTHA during the TACE procedure, has been reported because of its ability to offer relevant information for tumor identification, assessment of tumor feeding artery, and navigation guidance. ${ }^{22-24}$ Moreover, it has become possible for the accurate identification of tumor feeding arteries in combination with guidance software ${ }^{19}$, suggesting that CTHA will be performed more frequently during TACE. By performing not only the early phase of CTHA but also the late phase of CTHA and depicting the corona-like deep stain, CTHA can be used not only for tumor vessel identification and navigation guidance but also for determination of safety margin. As it requires two CT scans, in the early phase and in the delayed phase, it has the disadvantage of increasing the radiation exposure. However, since the therapeutic effect of TACE can be expected to increase, we consider that the benefits outweigh the risks for the patient. If conventional CT or cone-beam CT is performed before the end of the TACE procedure and accumulation of iodized oil to the corona enhancement area is insufficient, we believe that additional embolization should be performed considering the possibility of inadequate drug administration or the presence of the feeding artery. Whether the additional emboli- zation will improve the treatment effect is an issue for further study.

Our study had several limitations. First, our study population was small and the study was retrospective. This limitation could have led to some patient selection bias. Moreover, as many tumors with relatively small diameters have been enrolled and only four tumors were larger than $30 \mathrm{~mm}$ in diameter in this study, it is unclear whether the same results as this study apply to large diameter tumors. Therefore, confirmation of our findings would require additional studies on large numbers of patients, including patients with large diameter tumors. Second, the observation period after TACE was relatively short. Longer-term observations may increase local recurrence rates. However, there was a large difference in local recurrence rates between the two groups, and it is considered that there is a significant difference between the two groups even after long-term observation. Third, as late-phase CTHA was not performed at a single-slice level, assessment of corona enhancement area may be slightly incorrect. In the nodules in which local tumor recurrence was recognized despite the assessment that the embolization was obtained throughout the corona enhancement area, the corona enhancement area may have been underestimated.

In conclusion, local tumor recurrence was significantly lower when embolizing not only the tumor itself but also the corona enhancement area, and corona enhancement area may be an accurate safety margin in TACE. Our results suggested that TACE should be continued until the embolic area covers the entire corona enhancement area.

\section{References}

1. Forner A, Reig M, Bruix J. Hepatocellular carcinoma. Lancet 2018; 391: 1301-14. doi: 10.1016/S0140-6736(18)30010-2

2. Stefanini GF, Amorati P, Biselli M, Mucci F, Celi A, Arienti V, et al. Efficacy of transarterial targeted treatments on survival of patients with hepatocellular carcinoma. Cancer 1995; 75: 2427-34. doi: 10.1002/1097-0142(19950515)75:10<2427::aid-cncr2820751007>3.0.co;2-

3. Llovet JM, Bruix J. Systematic review of randomized trials for unresectable hepatocellular carcinoma: chemoembolization improves survival. Hepatology 2003; 37: 429-42. doi: 10.1053/jhep.2003.50047

4. Lo CM, Ngan H, Tso WK, Liu CL, Lam CM, Poon RT, et al. Randomized controlled trial of transarterial lipiodol chemoembolization for unresectable hepatocellular carcinoma. Hepatology 2002; 35: 1164-71. doi: 10.1053/ jhep.2002.33156

5. Llovet JM, Real MI, Monyana X, Planas R, Coll S, Aponte J, et al. Arterial embolization or chemoembolization versus symptomatic treatment in patients with unresectable hepatocellular carcinoma: a randomized controlled trial. Lancet 2002; 359: 1734-9. doi: 10.1016/S0140-6736(02)08649-X 
6. European Association for the Study of the Liver. EASL Clinical Practice Guidelines: management of hepatocellular carcinoma. J Hepatol 2018, 69: 182-236. doi: 10.1016/j.jhep.2018.03.019

7. Okusaka T, Okada S, Ueno H, Ikeda M, Shimada K, Yamamoto J, et al. Satellite lesions in patients with small hepatocellular carcinoma with reference to clinicopathologic features. Cancer 2002; 95: 1931-7. doi: 10.1002/ cncr.10892

8. Nakashima Y, Nakashima O, Tanaka M, Okuda K, Nakashima M, Kojiro M. Portal vein invasion and intrahepatic micrometastasis in small hepatocelIular carcinoma by gross type. Hepatol Res 2003; 26: 142-7. doi: 10.1016/ s1386-6346(03)00007-x

9. Miyayama S, Yamashiro M, Hashimoto $M$, Hashimoto $N$, Ikuno M, Okumura $\mathrm{K}$, et al. Comparison of local control in transcatheter arterial chemoembol zation of hepatocellular carcinoma $\leq 6 \mathrm{~cm}$ with or without intraprocedura monitoring of the embolized area using cone-beam computed tomography. Cardiovasc Intervent Radiol 2014; 37: 388-95. doi: 10.1007/s00270-0130667-2

10. Kittipitch B, Keerati H, Teeravut T, Teerha P. Safety margin of embolized area can reduce local recurrence of hepatocellular carcinoma after superselec tive transarterial chemoembolization. Clin Mol Hepatol 2019; 25: 74-85. doi: $10.3350 / \mathrm{cmh} .2018 .0072$

11. Kattipatanapong $\mathrm{T}$, Nishiofuku $\mathrm{H}$, Tanaka $\mathrm{T}$, Sato $\mathrm{T}$, Masada $\mathrm{T}$, Tatsumoto $\mathrm{S}$, et al. Improved local tumor control and survival rates by obtaining a 3D-safety margin in superselective transarterial chemoembolization for small hepatocellular carcinoma. Cardiovasc Intervent Radiol 2020; 43: 423-33. doi: 10.1007/s00270-019-02365-9

12. Ueda K, Matsui O, Kawamori Y, Nakamura Y, Kadoya M, Yoshikawa J, et al. Hypervascular hepatocellular carcinoma: evaluation of hemodynamics with dynamic CT during hepatic arteriography. Radiology 1998; 206: 161-6. doi: 10.1148/radiology.206.1.9423667

13. Ueda K, Matsui O, Kawamori Y, Kadoya M, Yoshikawa J, Gabata T, et al. Differentiation of hypervascular hepatic pseudolesions from hepatocellular carcinoma: value of single-level dynamic CT during hepatic arteriography. J Comput Assist Tomogr 1998; 22: 703-8. doi: 10.1148/radiology.206.1.9423667

14. Inoue E, Fujita M, Hosomi N, Sawai Y, Hashimoto T, Kuroda C, et al. Double phase CT arteriography of the whole liver in the evaluation of hepatic tumors. J Comput Assist Tomogr 1998; 22: 64-8. doi: 10.1097/00004728199801000-00011

15. Matsui O, Ueda K, Kobayashi S, Sanada J, Terayama N, Gabata T, et al. Intra- and perinodular hemodynamics of hepatocellular carcinoma: CT observation during intra-arterial contrast injection. Abdom Imaging 2002; 27: 147-56. doi: 10.1007/s00261-001-0091-y

16. Kitao A, Zen $Y$, Matsui $O$, Gabata T, Nakamura Y. Hepatocarcinogenesis: multistep changes of drainage vessels at CT during arterial portography and hepatic arteriography - radiologic-pathologic correlation. Radiology 2009; 252: 605-14. doi: 10.1148/radiol.2522081414

17. Sakon M, Nagano H, Nakamori S, Dono K, Umeshita K, Murakami T, et al. Intrahepatic recurrences of hepatocellular carcinoma after hepatectomy: analysis based on tumor hemodynamics. Arch Surg 2002; 137: 94-9. doi: 10.1001/archsurg.137.1.94

18. Sasaki A, Kai S, Iwashita Y, Hirano S, Ohta M, Kitano S. Microsatellite distribution and indication for locoregional therapy in small hepatocellular carcinoma. Cancer 2005; 103: 299-306. doi: 10.1002/cncr.20798

19. Miyayama S, Yamashiro M, Sugimori N, Ikeda R, Okimura K, Sakuragawa. Outcomes of patients with hepatocellular carcinoma treated with conventional transarterial chemoembolization using guidance software. J Vasc Interv Radiol 2019; 30: 10-8. doi: 10.1016/j.jvir.2018.08.009

20. Matsui O, Kobayashi S, Sanada J, Kouda W, Ryu Y, Kozaka K, et al Hepatocellular nodules in liver cirrhosis: hemodynamic evaluation (angiog raphy-assisted CT) with special reference to multi-step heptocarcinogenesis. Abdom Imaging 2011; 36: 264-72. doi: 10.1007/s00261-011-9685-1

21. Hirooka $M$, Ochi $H$, Koizumi $Y$, Tokumoto $Y$, Hiraoka $A$, Kumagi $T$, et al. Local recurrence of hepatocellular carcinoma in the tumor blood drainage area following radiofrequency ablation. Mol Clin Oncol 2014: 2: 182-6. doi: $10.3892 /$ mco. 2013.229
22. Takayasu K, Muramatsu Y, Maeda T, Iwata R, Furukawa H, Muramatsu Y, et al. Targeted transarterial oily chemoembolization for small foci of hepatocellular carcinoma using a unified helical CT and angiography system: analysis of factors affecting local recurrence and survival rates. Am J Roentgenol 2001; 176: 681-8. doi: 10.2214/ajr.176.3.1760681

23. Tacher V, Radaelli A, Lin M, Geschwind JF. How I do it: conebeam CT during transarterial chemoembolization for liver cancer. Radiology 2015; 274: 32034. doi: $10.1148 /$ radiol.14131925

24. Lee JH, Lee IJ, Kim HB, Park B, Kim BH, Park JW, et al. Efficacy and safety of transarterial chemoembolisation with cone-beam $\mathrm{CT}$ in patients with hepatocellular carcinoma within the Milan criteria: a retrospective cohort study. Clin Radiol 2019; 74: 407.e19-407.e28. doi: 10.1016/j.crad.2019.01.024

25. Chiaradia M, Izamis ML, Radaelli A, Prevoo W, Maleux G, Schlachter T, et al. Sensitivity and reproducibility of automated feeding artery detection software during transarterial chemoembolization of hepatocellular carcinoma. J Vasc Intervent Radiol 2018; 29: 425-31. doi: 10.1016/j.jvir.2017.10.025 